\title{
Una aproximación a la planificación en los archivos universitarios de Ecuador
}

\author{
Ana R. Pacios* \\ María José Bravo Ramos**
}

Artículo recibido:

30 de enero de 2021

Artículo aceptado:

6 de abril de 2021

Artículo de investigación

\section{Resumen}

Se presenta un análisis de la situación actual de la técnica de planificación en los archivos universitarios de Ecuador. Con el objetivo de conocer el uso y la utilidad de la planificación en estos archivos se elaboró una encuesta dirigida a las y los directores o responsables de un universo conformado por 58 archivos de las instituciones de educación superior ecuatorianas, tanto públicas como privadas, acreditadas por el Consejo Superior de Ecuador. La encuesta obtuvo una tasa de respuesta de $43 \%$. El análisis de los resultados de la encuesta muestra la necesidad de llevar a cabo acciones formativas en gestión y planificación de archivos

* Universidad Carlos III de Madrid, Departamento de Biblioteconomía y Documentación, España areyes@bib.uc3m.es

** Biblioteca General de la Escuela Politécnica Nacional, Ecuador

maria.bravor@epn.edu.ec

INVESTIGACIÓN BIBLIOTECOLÓGICA, vol. 35, núm. 88, julio/septiembre, 2021, México, ISSN: 2448-8321 pp. 127-145 
ante la falta de conocimientos para abordar la elaboración de un plan estratégico. Sólo un escaso número de archivos cuenta con planes estratégicos vigentes alineados a los planes institucionales pero, salvo excepciones, no han sido difundidos a través de sus sitios web.

Palabras clave: Archivos Universitarios; Planificación; Formación Archiveros; Ecuador

A review of university archive planning in Ecuador Ana R. Pacios and María José Bravo Ramos

\section{Abstract}

An analysis of the present situation of planning techniques by Ecuatorian university archives is described. The directors of the 58 archives kept by the country's private and public halls of higher education accredited by the High Council of Ecuador were surveyed, with a response rate of $43 \%$, to ascertain the use of planning and the utility of these institutions. The findings reveal a lack of strategic planning know-how and the concomitant need for archive management and planning training. With rare exceptions, none of the very few archives has up-to-date strategic plans in keeping with institutional aims and publish them on their websites.

Keywords: University Archives; Planning; Archivist Training; Ecuador

\section{INTRODUCCIÓN}

Fil 1 documento Propuesta de directrices para el diseño, consolidación y administración de Sistemas de Gestión de Documentos y Archivos de las Instituciones de Educación Superior de Ecuador (CES y RAUEC, 2016: 15) se refiere al archivo universitario como un "conjunto de documentos de cualquier fecha, formato o soporte material, producidos en el desarrollo de las funciones y actividades de los diferentes miembros universitarios con el objetivo de gestionar, conservar y difundir los documentos con finalidades administrativas, 
docentes, investigadoras y culturales de la universidad". Por su importante función, se ha visto una alta consideración hacia los archivos universitarios señalando que son fundamentales entre todos los recursos de información que custodia la universidad porque guardan documentos que son prueba tangible de su funcionamiento y constituyen su memoria histórica-documental (Rivas Fernández, 2004: 123; Tanodi, 2011: 17-18).

La gestión apropiada del archivo universitario repercute, fundamentalmente, en la mejora de la efectividad de la gestión general de la universidad. La gestión de una unidad de información como es un archivo requiere, al igual que una biblioteca, que sus profesionales cuenten con conocimientos en técnicas de Management o Administración. En particular, los relacionados con funciones como la dirección, planificación o comunicación (Cobo-Serrano y Arquero-Avilés, 2017: 248). Estas funciones ya fueron recogidas, entre otros, por Chacón Arias (1997) al indicar que, independientemente del nivel del archivo (final-intermedio-central), "requiere de excelentes administradores y obviamente, de una adecuada administración que facilite el logro eficiente de sus objetivos". De igual modo Alberch i Fugueras, Corominas Noguera y Martinez i Rodriguez (1997: 226-229) se refieren a estas funciones para los responsables de un archivo. La planificación, en este sentido, es una función vital en cuanto que ayuda a definir la dirección que debe tomar el archivo y los recursos necesarios para llegar allí, contribuyendo a su eficacia y eficiencia.

Toda planificación tiene que partir de las metas establecidas a nivel institucional para establecer las suyas propias, teniendo en cuenta el tamaño del archivo, el servicio, la tipología de los usuarios y los recursos disponibles, entre ellos el presupuesto. A pesar de las diferencias que puedan darse entre unos archivos y otros no debería prescindir ninguno de la planificación porque es un proceso flexible y adaptable a diferentes circunstancias (Arévalo Jordan, 1995: 41). Asimismo, Jardim (2010: 197-198), refiriéndose al caso iberoamericano, enfatiza en la necesidad de planificar para que sea viable la legislación archivística como parte estructural de la política archivística nacional, regional o local.

En relación con la archivística en América Latina, para el periodo 20002009 los trabajos publicados centrados en la administración del archivo como unidad responsable de la gestión documental son escasos y se relacionan con la calidad y los estudios de usuarios (Marín Agudelo, 2012: 88-90). La planificación parece haber quedado al margen en los estudios. La búsqueda en las bases de datos LISA y LISTA para el periodo 2010-2019 de trabajos sobre planificación en los archivos latinoamericanos, bajo esta misma perspectiva, no devuelve resultado alguno. 
Si quisiéramos acercarnos a la evolución de los archivos universitarios de Ecuador, la carencia de estudios es la nota dominante. La no edición de los sucesivos Congresos de Archivología de Mercosur, en los que suele haber una reunión de archivos universitarios, impide la difusión de cualquier avance, problema o desafío a los que se enfrentan este tipo de archivos en Latinoamérica y, en particular, en cada país. Tampoco ha sido objeto de interés en obras de conjunto recientes sobre archivos de América Latina (ej. Porras P. y Zúñiga M., 2019).

Sin embargo, desde inicios del siglo XXI, se evidencia que el Consejo de Educación Superior de Ecuador (en adelante, CES), preocupado por la recuperación, organización y consolidación de la memoria y patrimonio documental de las Instituciones de Educación Superior (IES), ha emprendido acciones y propuesto directrices para su buen funcionamiento. Una de ellas ha sido la elaboración de un diagnóstico de la situación así como la formación y capacitación profesional de su personal, en especial de los responsables del archivo (CES y RAUEC, 2016: 6). En 2015 se crea la Red de Archivos Universitarios del Ecuador (RAUEC) con el fin de desarrollar acciones que conduzcan a la implentación de estándares de la gestión documental y el tratamiento archivístico de los documentos. En su sede web se muestran los grupos estratégicos de trabajo (https://sites.google.com/view/rauec/archivos-universitarios).

Las directrices se dirigen a los funcionarios responsables de la gestión documental en las universidades del Ecuador, para que puedan diseñar, planificar y consolidar Sistemas de Gestión de Documentos y Archivos Universitarios (CES y RAUEC, 2016: 4). La planificación se revela, por tanto, como un elemento imprescindible para fijar líneas de actuación y estrategias para el desarrollo de la actividad de los archivos ecuatorianos.

Por lo que se refiere a los cuatro diagnósticos de todos los archivos institucionales del país llevados a cabo entre 2000 y 2011, han permitido visualizar una situación grave al poner de manifiesto que más de $70 \%$ de los archivos estaban en peligro de deterioro, y que en más de $40 \%$ de éstos, no se ejecutaban normas técnicas para la organización de los fondos documentales que custodiaban (CES y RAUEC, 2016: 5). Es evidente que algunos han puesto en marcha métodos de trabajo que se han convertido en ejemplos de buenas prácticas, aunque no se conoce el alcance real de las medidas implementadas.

Interesa destacar el diagnóstico resultado de la Encuesta Nacional de Situación de Archivos Universitarios que se llevó a cabo en 2014 para conocer la situación de los archivos universitarios del país y a la que contestaron 49 instituciones de un total de 56 (Franco Suárez y Camacaro, 2016). En 45 \% de las instituciones el archivo no se evidencia como unidad administrativa centralizada por lo que no presta ningún tipo de servicio. De las 56 instituciones, 30 
registraron que sí tenían Archivo Central y 26 que no. Un resumen de algunos aspectos destacados que se recogen en el estudio citado se anota a continuación por ser el trabajo más reciente que da muestra de la situación. Acerca de sus funciones, un importante número de universidades respondió que tiene más relevancia la salvaguarda de la información, en contraposición a su difusión, actividad que se ubicó en último nivel de importancia; la consulta y el préstamo de información son los principales servicios que prestan. Sobre su infraestructura tecnológica, $50 \%$ de universidades no contaba con ninguna para realizar tareas de procesamiento técnico de la información y el 40 \% no posee ningún tipo de software de archivo. Treinta y cinco archivos no ejecutaban ninguna actividad de procesamiento técnico centralizado, lo que refleja claramente un alto nivel de ausencia de parámetros de control de los sistemas de gestión documental. En lo referente al personal, 80 \% presentaba problemas de personal especializado en el campo de la archivística y 73 \% no poseía personal técnico especializado. Una encuesta posterior realizada en 2016, centrada en el talento humano, mostró un cambio en la situación debido al Programa de Formación Continua en Archivología y Gestión de la Información, que permitió al personal acceder a conocimientos técnicos actualizados.

No se localizó trabajo alguno que evidencie los resultados de la implementación de estos programas de formación derivados de las deficiencias detectadas en las encuestas y tendentes a garantizar la buena gestión de los archivos y su fondo documental. Es un proceso a medio y largo plazo que sin duda tendrá que dar resultados.

Ante la situación y el tiempo transcurrido desde las encuestas aludidas, este trabajo se planteó dar respuesta a la siguiente pregunta de investigación: ¿se está haciendo uso de la planificación estratégica en los archivos universitarios de Ecuador como herramienta para diseñar estrategias y cursos de acción adecuados que permitan el logro de objetivos en estos servicios?

\section{Metodología}

Con el objetivo de conocer el uso y utilidad de la planificación en los archivos universitarios ecuatorianos se llevó a cabo una encuesta dirigida a sus responsables. Se eligieron los 58 archivos universitarios dependientes del mismo número de instituciones de Educación Superior, tanto públicas como privadas, acreditadas por el Consejo Superior de Ecuador (Anexo I).

La encuesta se configuró en Google Forms y su URL de acceso se envió a través del correo electrónico de cada director/a o responsable, recopilados a través de la sede web de la RAUEC. Se recurrió a esta institución para 
conseguir los contactos porque son archivos con apenas visibilidad en la sede web de la universidad a la que pertenecen. Sólo siete archivos tienen página web, cinco de los cuales cuentan con una página informativa sobre sus servicios, y los dos restantes disponen de un catálogo para consulta de información.

En el texto del correo se explicaba el objetivo de la encuesta y el marco del trabajo de investigación en el que se hacía. Fue enviada durante el periodo del 4 de octubre al 11 de noviembre de 2019 y se hicieron dos recordatorios cuando no se contestó en el plazo establecido.

La encuesta estaba formada por 33 preguntas, combinando las dicotómicas y abiertas, agrupadas en tres secciones (Anexo II):

- La primera, de 15 preguntas, pretendía recoger los datos del responsable o director del archivo (contacto, sexo, edad, denominación del cargo, años de experiencia, tipo de archivo en el que ejercía su actividad, número de personas integrantes del equipo y formación recibida, en particular la relacionada con las funciones de administración y planificación).

- La segunda, con seis preguntas, perseguía conocer cuestiones relacionadas con la planificación (el interés de la técnica para el archivo, si había experiencia previa en la aplicación de la misma y si existía un plan).

- La tercera contenía 12 preguntas sólo para aquellos que hubieran contestado afirmativamente a la existencia de un plan en el archivo, incluyendo la que posibilitaba hacer cualquier comentario o sugerencia que estaba abierta a cualquier director/a o responsable de archivo.

\section{Presentación y ANÁlisis de Resultados}

La encuesta fue respondida por 25 archiveros/as siendo la tasa de respuesta de $43 \%(n=58)$. A continuación se exponen los resultados atendiendo a las secciones de la misma.

\section{Perfil organizacional de los archivos universitarios y de su personal}

La respuesta a la primera sección nos permite hacer el siguiente perfil de los profesionales y el tipo de archivo que dirigen: $41.7 \%$ son hombres mientras que $58.3 \%$ son mujeres; la edad de los archiveros oscila entre los 27 y los 69 años, siendo la edad media de 43 años; los años de experiencia en el cargo de dirección está entre uno y 37, con una media de 12 años. Por lo que se refiere 
a la plantilla con la que cuentan estos archivos, está integrada por entre dos y 15 personas, siendo la media de cuatro. Respecto al tipo de archivo universitario existente en las universidades ecuatorianas las respuestas indican que $13 \%$ son históricos, $17.4 \%$ son intermedios y $69.6 \%$ son archivos centrales. Con estos datos se evidencia que los archivos universitarios en los que ejercen su actividad estos profesionales son, en su mayoría, centrales. Respecto a las preguntas cerradas relacionadas con las funciones y responsabilidades, las respuestas indicaron lo siguiente: 66.7 \% respondió que el archivo las tenía claramente definidas, $25 \%$ que solamente estaban definidas algunas funciones y $8.3 \%$ restante no tenía definidas ni las funciones ni las responsabilidades de cada puesto.

Otro aspecto de gran relevancia es la formación profesional de los/as directores/as del archivo. Sus respuestas permitieron identificar que tienen conocimientos en materias muy diversas al ser titulados en distintas áreas: administración de empresas y marketing, ciencias de la educación, archivología, periodismo, gestión de la formación, derecho, política y gestión de la innovación tecnológica, economía, informática y sistemas, comunicación y educación audiovisual, psicología.

En particular, se preguntaba por la formacion específica en administración y planificación de archivos. Respecto a la primera, $79.2 \%$ de directores/ as sí habían recibido formación en administración de archivos mientras que 20.8 \% restante no contaban con formación en esta temática. Respecto a la segunda, $58.3 \%$ de directores/as confirmaron que habían recibido formación específica en planificación de archivos (de los cuales $35.7 \%$ lo hicieron en la carrera universitaria y 85.7 \% a través de cursos especializados, lo que implica que 21.4 \% adquirió la formación por las dos vías), mientras que 41.7 \% no cuenta con ninguna formación específica en dicha temática.

Por lo que se refiere al resto del personal del archivo, los responsables manifestaron que $37.5 \%$ se ha capacitado en este ámbito mientras que $62.5 \%$ restante no lo ha hecho.

\section{Uso e importancia de la planificación}

En la segunda sección, específica sobre la planificación, las preguntas muestran los resultados que se anotan a continuación.

Respecto a la importancia de esta técnica, todos los directores/as la consideran totalmente relevante para que el archivo preste servicios de calidad. Sobre el tipo de planificación que se ha llevado a cabo en estos archivos, las respuestas ponen de manifiesto que son los proyectos los que ocupan el primer lugar, seguidos de los planes operativos anuales y los planes estratégicos (Tabla 1). 


\begin{tabular}{|l|r|}
\hline \multicolumn{1}{|c|}{ Denominación } & Número \\
\hline Plan estratégico (3 a 5 años) & 7 \\
\hline Plan operativo anual & 8 \\
\hline Proyectos & 9 \\
\hline Programas & 3 \\
\hline Otros & 3 \\
\hline Total & 30 \\
\hline
\end{tabular}

Tabla 1. Tipos de planes en los archivos universitarios

Los archivos que no hacen uso de la planificación justificaron este comportamiento argumentando las siguientes razones: falta de formación (dos), falta de tiempo (dos), no lo considera necesario (uno) y la falta de apoyo por parte de las autoridades (uno).

\section{Existencia de planes estratégicos}

En la tercera sección, relativa a la existencia de planes estratégicos en los archivos, las respuestas indicaron lo siguiente (Figura 1): $62.4 \%$ de los archiveros/as manifestaron que no lo tenían pero que se encontraban en proceso de elaboración, 16.7 \% contaba con un plan estratégico cuyo periodo de vigencia está comprendido entre uno y cinco años, otro $16.7 \%$ no cuenta con plan alguno y $4.2 \%$ expresaron que el plan había vencido.

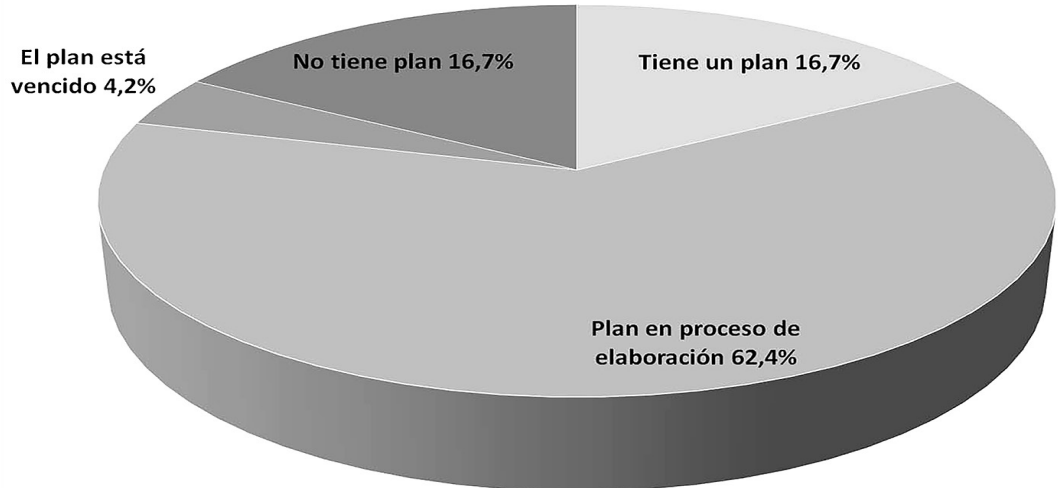

Figura 1. Situación de los planes estratégicos Fuente: elaboración propia 
Sobre los motivos por los que el archivo no contaba con un plan estratégico se dieron los mismos argumentos que en la pregunta sobre la razón por la que no se hacía uso de la planificación.

En relación con la pregunta sobre la difusión del plan a través de la sede web del archivo, las respuestas indicaron que $65 \%$ no cuentan con un sitio web propio del archivo, $25 \%$ manifiesta que el plan no está publicado y sólo $10 \%$ lo ha publicado en el sitio web. Los motivos argumentados por los que el plan no estaba disponible en la web fueron los siguientes, a pesar de que la pregunta iba sólo dirigida a los que tenían un plan: no se disponía de un sitio, el plan estaba en elaboración, porque sigue el plan institucional; se informa del mismo a través de una publicación institucional que lo hace de todos los proyectos, la universidad no cuenta con archivo.

Dada la conveniencia de conocer si al hacer el plan estratégico se había tenido en cuenta el institucional, se preguntó por esta cuestión obteniendo el siguiente resultado: $77.8 \%$ manifestaron que sí lo tuvieron en cuenta, 16.7 \% indicó que no lo había tenido en cuenta porque la institución no tenía plan alguno y 5.6 \% restante respondió que no consideraron importante vincular el plan del archivo con el de la institución.

Respecto al uso de herramientas para planificar, dada la posibilidad de respuesta múltiple, los archivos señalaron que están haciendo uso de indicadores (11), de la matriz FODA (10), del Cuadro de Mando Integral (tres) y otros (cinco) como sistemas propios elaborados por los departamentos, reglas técnicas, etc.

La implementación de los planes se estaba llevando a cabo conforme a lo previsto en $62.5 \%$ de los archivos mientras que $37.5 \%$ restante no lo hace. Esta última circunstancia se debe a diferentes causas, entre las que destacan la insuficiencia presupuestaria y la falta de espacio adecuado para el archivo.

El seguimiento de los planes se realiza por parte de $58.8 \%$ de los archivos y $35.3 \%$ restante no lo hace. El 5.9 \% indicó que sólo lo hace a veces. El período fijado para hacer el seguimiento varía desde los que lo hacen conforme a lo establecido en los objetivos (seis), anualmente (uno), mensualmente (tres), a la finalización del plan (uno), hasta aquellos que sólo lo hacen cuando disponen de tiempo (dos).

Finalmente, acerca del beneficio que supone para el archivo la elaboración y puesta en marcha de un plan estratégico, 76.9 \% de los responsables indicó que era grande mientras que para $23.1 \%$ era suficiente. Ninguno señaló como opciones poco o nada beneficioso. 


\section{Discusión}

Los resultados de este diagnóstico ponen de relieve deficiencias en algunos aspectos, aunque no todas son extensibles a todos los archivos:

- Necesidad de definir las funciones y responsabilidades de los puestos de trabajo, dado que $25 \%$ manifestaron que solamente tenían algunas y $8.3 \%$ ninguna. El catálogo de puestos de trabajo recoge el contenido de cada puesto de trabajo y los identifica de forma que muestra las funciones de cada uno de los distintos tipos de profesionales existentes en el archivo del que se trate (CAU-CRUE, 2004; Sanchis Moreno, 2009).

- Falta de formación en técnicas de planificación, según se desprende de la respuesta dada por $41.7 \%$ de los directores que admitieron no contar con formación específica en la misma. La formación profesional de los/as directores/as de archivo en el campo de la archivología y la gestión documental sigue siendo muy limitada pese a los esfuerzos realizados a través del Programa de Gestión de la Memoria de la Educación Superior, capacitación para los archiveros de las IES promovido por el CES en 2014 que respondía a las realidades concretas de las universidades ecuatorianas.

El interés por la planificación en los archivos no es nuevo. Prozor (1990) ya destacó su interés en los años 90. Boadas i Raset (2006: 455) manifiesta que:

cualquier servicio de archivo, por falto de recursos y aparentemente modesto que sea, debería elaborar su plan estratégico en donde expresar de manera detallada y sistemática cuáles son sus objetivos, en el espacio temporal que se haya fijado previamente, qué políticas y actuaciones tiene previstas para conseguirlos, y los sistemas e instrumentos de control correspondientes que permitan la evaluación de los resultados obtenidos.

En el ámbito de los archivos universitarios, por ejemplo, en España se han llevado a cabo iniciativas para favorecer el proceso de planificación, en particular la elaboración de planes estratégicos (CAU-CRUE, 2011), ayudando a tomar decisiones sobre la dirección que el archivo quiere seguir para alcanzar resultados, lo que demuestra que los planes estratégicos son una apuesta de futuro para reforzar su presencia en la universidad.

En el momento de redactar este trabajo (diciembre de 2020), la escasa oferta académica archivística en Ecuador se vincula con dos universidades: 
- Universidad Andina Simón Bolívar, sede Ecuador, con un programa de Maestría de Archivística y Sistemas de Gestión Documental, además de cursos de archivística.

- Universidad Técnica de Manabí, en donde se puede estudiar la licenciatura en Bibliotecología, Archivística y Documentación y el programa de maestría en Bibliotecología, mención Gestión de la información.

La Universidad de Guayaquil contó con la licenciatura de Bibliotecología y Archivología hasta 2020, lo que se deduce de los trabajos de su repositorio, pero ha dejado de ofertarla.

Además, desde la Asociación Ecuatoriana de Archiveros se imparten cursos, charlas y webinars sobre temas relacionados con la Gestión Documental y la Archivística.

- Falta de visión a largo plazo o estratégica, detectada a raíz de los escasos planes estratégicos con los que cuentan estos servicios (sólo en siete de ellos), a pesar de que la mayoría de los profesionales valora el beneficio de contar con un plan. La planificación a través de proyectos es lo más común en cuanto a temporalidad, pero se requiere que el archivo identifique y analice factores externos al mismo y trate de igualarlos con las capacidades que tiene como organización para hacer frente a los cambios y optimizar recursos (Navajo Gómez, 2009: 28), además de visualizar nuevas oportunidades y amenazas. Y esto sólo es posible mediante un proceso de planificación estratégica.

- Necesidad de difundir los planes de los archivos entre los miembros de la comunidad universitaria a través de diferentes vías. En el momento en que se hizo la encuesta resulta algo inusual al carecer el archivo de sede web propia, según manifestaron.

Como elemento a su favor, y a pesar de los pocos planes existentes, cabe mencionar que han sido desarrollados considerando el plan estratégico u otros planes de la institución a la que el servicio de archivo está adscrito, lo cual es un indicio de que están alineados a los objetivos y estrategias de la universidad. Esto está en la línea de lo argumentado por Arévalo Jordan (1995: 224), al aseverar que los archivos deben planificar acorde al plan general de la institución de forma permanente y ajustándose a sus políticas para posibilitar la consecución de los objetivos y metas del servicio de archivo. 
La respuesta a la pregunta de investigación formulada en este trabajo es que los archivos universitarios de Ecuador apenas utilizan la planificación estratégica como técnica básica para enfrentarse a su futuro. Sólo si los archivos planifican pueden hacer una evaluación crítica de sí mismos, confeccionar un equilibrio realista entre las situaciones reales y el objetivo que persiguen y así generar argumentos convincentes para justificar sus demandas y objetivos (Zittel, 1987: 217).

Los archiveros, al igual que otros profesionales de la información (bibliotecarios, gestores de la información, etc.), necesitan adquirir el mismo tipo de competencias para poder gestionar su servicio con eficacia y eficiencia. De ahí que la administración haya sido considerada un área clave en la formación de cualquiera de estos especialistas, como prueban los intentos de armonizar a nivel internacional el curriculum de Management por parte de la FID, ICA e IFLA desde bien temprano (Vaughan, 1987: i). En particular, la competencia relacionada con la planificación se ha venido insertando, en el ámbito latinoamericano, en la gestión o administración de sistemas, instituciones y servicios archivísticos (Marín Agudelo, 2013:306) aunque no se puede generalizar esta afirmación para todos los países si se tiene en cuenta los reducidos programas existentes enfocados en la archivística. El déficit evidente de programas de archivística en América Latina sin duda tiene que ver con las carencias formativas detectadas en este trabajo; en Ecuador, particularmente, donde la falta de planes de estudio se ha puesto en evidencia en diferentes ocasiones (ej. Mendoza Navarro, 2008). Es imposible abordar el diseño de estrategias administrativas y sistemas institucionales de archivos que se exige a los archiveros (De Vega, 2015: 5) sin tener conocimiento de la función de planificación y de sus técnicas asociadas. Lo mismo sucede cuando se enfrentan a la toma de decisiones y a la formulación de políticas públicas.

A la espera de que se amplíen los escasos programas formativos de archivística en Ecuador, se confía en que las iniciativas del CES permitan al personal de estos archivos diseñar, planificar, consolidar y evaluar estos servicios como oportunidad viable para iniciar procesos de mejora de las funciones que desempeñan. 


\section{REFERENCIAS}

Alberch i Fugueras, Ramón, Mariona Corominas Noguera y Maria Carme Martinez i Rodriguez. 1977. "El personal dels arxius. La funció arxivística i la seva repercussió en la plantilla”. Lligall: revista catalana d'Arxivística 11: 221-252.

Arévalo Jordán, Víctor Hugo. 1995. Planificación general de archivos. Santa Fe: Santa Fe.

Boadas i Raset, Joan. 2006. "Archivos: Planificar, gestionar, actuar!”, en Archivo, ¿un servicio público?, Actas I Congreso de Archivos de Canarias (La Oliva, Fuerteventura, 19-21 de octubre), 421-468. Las Palmas de Gran Canaria: Anroart. Fecha de consulta: septiembre 2020. https://www.girona.cat/sgdap/docs/Archivos_planificar.pdf

CAU (Conferencia de Archiveros de las Universidades Españolas), CRUE. 2004. Catálogo de funciones del personal de los archivos universitarios. Fecha de consulta: noviembre 2020.

http://cau.crue.org/wp-content/uploads/RRHHcatalogovalladolid2004.pdf

CAU-CRUE. 2011. Planificación Estratégica de Archivos Universitarios. Guía para la elaboración de un plan estratégico para archivos universitarios. Fecha de consulta: noviembre 2020.

http://cau.crue.org/wp-content/uploads/guia_plan_estrategico_2011.pdf

CES y RAUEC (Consejo de Educación Superior y Red de Archivos Universitarios del Ecuador). 2016. Propuesta de directrices para el diseño, consolidación y administración de Sistemas de Gestión de Documentos y Archivos de las Instituciones de Educación Superior. Quito: Consejo de Educación Superior.

Chacón Arias, Virginia. 1997. "Funciones, objetivos y prioridades de una moderna y científica administración y legislación de archivos", en Proceedings of the 13th. International Congress on Archives (Beijing, 2-7september 1996). Archivum XLIII: 168-177.

Cobo-Serrano, Silvia y Rosario Arquero-Avilés. 2017. "La gestión de proyectos en las bibliotecas universitarias: percepciones de los profesionales latinoamericanos”. Revista General de Información y Documentación 27 (1): 247-260.

De Vega, Mercedes. 2015. "Archivos y formación académica en América Latina”, en I Congreso Internacional de Archivística: Sistemas y Redes en el Ecuador. Fecha de consulta: noviembre 2020. https://issuu.com/ricardoblanco15/docs/archivos_y_formacion_academica_en_a

Franco Suárez, Priscila y María Emilia Camacaro. 2016. "Encuesta Nacional de Situación de Archivos Universitarios 2014-2015 y Encuesta de Profesionalización de Archivos Universitarios 2016”, en Memorias Primer Encuentro Nacional de Archivos Universitarios. Gestión de la Información y Memoria Archivística: Estado de situación, desafíos y perspectivas (Quito, julio 2016), 31-47. Quito: Consejo de Educación Superior CES. Fecha de consulta: septiembre 2020. https://drive.google.com/file/d/1lqY-qXioiMY0ZFSLXUVdJicO6OiSm63s/view

Jardim, José Maria. 2010. Políticas y sistemas de archivos. México: Secretaría General Iberoamericana, Red de Archivos Diplomáticos Iberoamericanos.

Marín Agudelo, Sebastián Alejandro. 2012. "Estado de la archivística en América Latina 2000-2009. Perspectivas teóricas y aproximaciones conceptuales”. Investigación Bibliotecológica 26 (57): 77-101.

http://dx.doi.org/10.22201/iibi.0187358xp.2012.57.33840 
Marín Agudelo, Sebastián Alejandro. 2013. "Formación Archivística en América Latina: Una revisión de los perfiles y las competencias”. Revista Interamericana de Bibliotecología 35 (3): 299-309. https://revistas.udea.edu.co/index.php/RIB/article/view/15877

Mendoza Navarro, Aida Luz. 2008. "Hacia la homologación de los planes de estudios de archivística en América Latina”. Revista d'Arxius 7: 137-194. Fecha de consulta: septiembre 2020.

http://arxiversvalencians.org/wp-content/uploads/2020/04/revista2008_mendoza.pdf

Navajo Gómez, Pablo. 2009. Planificación estratégica en organizaciones no lucrativas. Guia participativa basada en valores. Madrid: Narcea.

Porras P., María Elena y Daniela Zúñiga M., eds. 2019. Archivística sin fronteras: Reflexiones sobre politicas de gestión, formación e investigación en archivos. Quito: Consejo de la Judicatura, Archiveros sin Fronteras, Universidad Andina Simón Bolívar. Fecha de consulta: septiembre 2020. http://repositorio.uasb.edu.ec/bitstream/10644/7424/1/Porras-Zu\%c3\%b1iga-Archivistica.pdf

Prozor, Novi. 1990. La gestion des archives et des documents au service des décideurs: Une étude RAMP. UNESCO. Fecha de consulta: septiembre 2020.

http://www.nzdl.org/gsdlmod?e=d-00000-00---off-0tulane--00-0----0-10-0---0--0direct-10---4-------0-11--11-en-50---20-help---00-0-1-00-0--4---0-0-11-10-0utfZz-8$00 \& \mathrm{a}=\mathrm{d} \& \mathrm{c}=$ tulane $\& \mathrm{cl}=\mathrm{CL} 1.3 \& \mathrm{~d}=$ HASH019a945757df59d4fbdac 514.3

Rivas Fernández, José Bernal. 2004. "Los archivos universitarios. El procesamiento de sus fondos documentales y la difusión de sus contenidos". Reflexiones 83 (1): 121-129. Fecha de consulta: septiembre 2020. https://revistas.ucr.ac.cr/index.php/reflexiones/article/view/11390/10745

Sanchis Moreno, Francisco. 2009. Informe sobre un catálogo de funciones y tareas del personal archivero. Fecha de consulta: septiembre 2020. http://www.arxiversvalencians.org/wp-content/uploads/2016/05/informe_funcions_i_tasques_del_personal_arxiver.pdf

Tanodi, Branka. 2011. "El archivo universitario, algunas reflexiones". Anuario Escuela de Archivología III: 16-23. Fecha de consulta: septiembre 2020 https://revistas.unc.edu.ar/index.php/anuario/article/view/12640

Vaughan, Antony, comp. 1987. International reader in the management of library, information and archive for the General Information Programme and UNISIST. Paris: Unesco.

Zittel, Bernard. 1987. "Archive planning", en International reader in the management of library. Information and archive services, Anthony Vaughan, comp., 215-225. Paris: Unesco. Fecha de consulta: septiembre 2020. https://unesdoc.unesco.org/ark:/48223/pf0000079856

Para citar este texto:

Pacios, Ana R. y María José Bravo Ramos. 2021. "Una aproximación a la planificación en los archivos universitarios de Ecuador". Investigación Bibliotecológica: archivonomía, bibliotecología e información 35 (88): 127-145. 
Anexo I. Universidades a las que se envió la encuesta con indicación de las que respondieron a la misma

\begin{tabular}{|c|c|}
\hline Universidades & Respuesta \\
\hline Escuela Politécnica Nacional & - \\
\hline Escuela Superior Politécnica Agropecuaria de Manabí & $X$ \\
\hline Escuela Superior Politécnica de Chimborazo & - \\
\hline Escuela Superior Politécnica del Litoral & - \\
\hline Facultad Latinoamericana de Ciencias Sociales & $x$ \\
\hline Instituto de Altos Estudios Nacionales & $x$ \\
\hline Pontificia Universidad Católica del Ecuador & $x$ \\
\hline Universidad Agraria del Ecuador & - \\
\hline Universidad Andina Simón Bolívar & $x$ \\
\hline Universidad Casa Grande & $x$ \\
\hline Universidad Católica de Cuenca & $x$ \\
\hline Universidad Católica de Santiago de Guayaquil & - \\
\hline Universidad Central del Ecuador & - \\
\hline Universidad de Cuenca & - \\
\hline Universidad de Especialidades Turísticas & - \\
\hline Universidad de las Américas & - \\
\hline Universidad de las Artes & $x$ \\
\hline Universidad de las Fuerzas Armadas (ESPE) & - \\
\hline Universidad de los Hemisferios & $x$ \\
\hline Universidad de Otavalo & $x$ \\
\hline Universidad del Azuay & - \\
\hline Universidad del Pacífico Escuela de Negocios & - \\
\hline Universidad Estatal Amazónica & - \\
\hline Universidad Estatal de Milagro & - \\
\hline Universidad Estatal de Bolívar & $x$ \\
\hline Universidad Estatal del Sur de Manabí & $x$ \\
\hline Universidad Estatal Península de Santa Elena & - \\
\hline Universidad Iberoamericana del Ecuador & - \\
\hline Universidad Internacional del Ecuador & - \\
\hline Universidad Laica Eloy Alfaro de Manabí & - \\
\hline Universidad Laica Vicente Rocafuerte de Guayaquil & - \\
\hline Universidad Metropolitana & - \\
\hline
\end{tabular}




\begin{tabular}{|c|c|}
\hline Universidad Nacional de Chimborazo & $x$ \\
\hline Universidad Nacional de Loja & $x$ \\
\hline Universidad Particular de Especialidades Espíritu Santo & - \\
\hline Universidad Particular Internacional Sek & - \\
\hline Universidad Particular San Gregorio de Portoviejo & - \\
\hline Universidad Regional Amazónica Ikiam & - \\
\hline Universidad Regional Autónoma de los Andes & - \\
\hline Universidad San Francisco de Quito & - \\
\hline Universidad Santiago de Guayaquil & $x$ \\
\hline Universidad Politécnica Estatal del Carchi & $x$ \\
\hline Universidad Politécnica Salesiana & $x$ \\
\hline Universidad Técnica de Ambato & $x$ \\
\hline Universidad Técnica de Babahoyo & $x$ \\
\hline Universidad Técnica de Cotopaxi & $x$ \\
\hline Universidad Técnica de Machala & $x$ \\
\hline Universidad Técnica de Manabí & - \\
\hline Universidad Técnica del Norte & $x$ \\
\hline Universidad Técnica Estatal de Quevedo & - \\
\hline Universidad Técnica Luis Vargas Torres de Esmeraldas & - \\
\hline Universidad Técnica Particular de Loja & $x$ \\
\hline Universidad Tecnológica Ecotec & - \\
\hline Universidad Tecnológica Empresarial de Guayaquil & - \\
\hline Universidad Tecnológica Indoamérica & $x$ \\
\hline Universidad Tecnológica Israel & - \\
\hline Universidad UTE (Tecnológica Equinoccial) & $x$ \\
\hline Yachay Tech & - \\
\hline
\end{tabular}

Anexo II. Encuesta enviada a los responsables de los archivos universitarios

\section{La planificación en los archivos universitarios ecuatorianos}

\section{Datos personales, de formación y archivo}

1. Nombre y apellidos:

2. Sexo: 
3. Edad:

4. Universidad a la que pertenece:

5. Según la clasificación de archivos, ¿cuál de los siguientes administra usted?

[ ] Archivo central

[ ] Archivo intermedio

[ ] Archivo histórico

6. Nombre del archivo que administra:

7. Cargo que desempeña:

8. Años de experiencia en el cargo:

9. Título que ostenta:

10. Número de personas que conforman la plantilla del archivo:

11. ¿Están claramente definidas las funciones y responsabilidades del personal del archivo en las diferentes áreas?

[ ] Sí

[ ] Algunas

[ ] No

12. ¿Ha recibido formación en administración de archivos?

[ ] Sí

[ ] No

13. ¿Ha recibido formación específica en planificación de archivos?

[ ] Sí

[ ] No

14. Si la respuesta anterior es positiva, indique la forma:

[ ] En la carrera universitaria

[ ] A través de cursos especializados

15. ¿Considera que el personal archivero a su cargo está formado en materia de planificación?

[ ] Sí

[] No

\section{Planificación}

16. ¿Considera que la planificación es importante para que el archivo preste servicios de calidad?

[ ] Sí

[ ] No

[ ] No tiene relación la calidad de los servicios con la planificación

17. ¿Ha planificado alguna vez en el archivo que usted administra?

[ ] Sí

[ ] No 
18. Si la respuesta a la pregunta anterior fue positiva ¿qué tipo de planificación se ha llevado a cabo?

[ ] Plan estratégico (de 3 a 5 años)

[ ] Plan operativo anual

[ ] Proyectos

[ ] Programas

[ ] Otra:

19. Si la respuesta a la pregunta 17 fue negativa, indique las razones:

20. Al momento, ¿cuenta el archivo con un plan estratégico vigente?

[ ] Sí

[ ] En proceso de elaboración

[ ] Está vencido

[ ] No

21. En caso de NO contar con un plan estratégico, indique los motivos:

\section{El plan estratégico}

22. ¿Se ha difundido el plan al personal del archivo?

[ ] Sí

[ ] No

[ ] Sólo lo conoce el personal involucrado en la administración del archivo

23. En caso de que el archivo cuente con una página web, ¿el plan está publicado en este sitio?

[ ] Sí

[ ] No

[ ] No existe un sitio web propio del archivo

24. En caso de que el plan no esté difundido, indique las razones por las cuales no se ha hecho:

25. En la elaboración del plan ¿se tuvo en cuenta el plan estratégico u otros planes de la institución?

[ ] Sí

[ ] No, porque la institución no cuenta con ningún plan

[ ] No tengo conocimiento de la existencia de ningún plan institucional

[ ] No considero que sea importante vincular el plan del archivo con el de la institución

26. ¿Qué herramientas utilizó para planificar los objetivos y estrategias del archivo?

[ ] Cuadro de mando de integral

[ ] Matriz FODA 
[ ] Indicadores

[ ] Ninguna

[ ] Otra:

27. Indique el plazo o período de años que comprende el plan estratégico que tiene el archivo:

28. ¿Se está implementando el plan estratégico conforme a lo establecido en el mismo?

[ ] Sí

[ ] No

29. En caso de no implementarse el plan, indique las razones por las que no se hace:

30. ¿Se hace un seguimiento del plan estratégico?

[ ] Sí

[ ] A veces

[ ] No

31. ¿Cada cuánto tiempo se hace el seguimiento del plan a fin de detectar posibles desviaciones?

[ ] Según el plazo de los objetivos fijados

[ ] Anualmente

[ ] Cuando se dispone de tiempo para hacerlo

[ ] Mensualmente

[ ] A la finalización del plan

32. ¿Considera que el plan estratégico del archivo beneficia a las estrategias y objetivos de la universidad?

[ ] Mucho

[ ] Lo suficiente

[ ] Poco

[ ] Nada

33. Añada cualquier sugerencia o comentario que considere oportuno para completar o aclarar la información de la encuesta: 